\title{
Porcine circovirus-like agents in sera of dogs and cats in China
}

\author{
libin wen ${ }^{1}$, aihao mao ${ }^{1}$, jianping $x^{1}{ }^{1}$, and kongwang he ${ }^{1}$ \\ ${ }^{1}$ Jiangsu Academy of Agricultural Sciences
}

May 8, 2020

\begin{abstract}
Porcine circovirus-like agents comprise two types of viruses: porcine circovirus-like viruses (P1, P2, P3, and P4) and porcine circovirus-like mini agents (PCVL258, PCVL264, PCVL201, and PCVL347). Of these, P1 has been identified in pigs, cattle, goats, and rabbits in China; P2, P3, P4, PCVL258, and PCVL264 have been identified in pigs; and PCVL201 and PCVL347 have been identified in cattle. The purpose of this study was to determine whether dogs and cats have been exposed to porcine circovirus-like agents. We screened 158 serum samples from diseased dogs and 41 from cats in China by PCR and nucleotide sequencing. In dogs, approximately $18 \%(\mathrm{n}=28)$ were positive for P1, 17\% $(\mathrm{n}=26)$ for PCVL258, and 9\% ( $\mathrm{n}=14)$ for PCVL264; in cats, $17.1 \%(n=7)$ were positive for $\mathrm{P} 1,9.8 \%(\mathrm{n}=4)$ for $\mathrm{P} 4$, and $14.6 \%(\mathrm{n}=6)$ for PCVL258. The P1 genomes in this study consisted of 648 nucleotides (nt), and shared 96.8 to $100 \%$ nt identity with other P1 genomes in GenBank. The P4 genome shared 98.3 to $100 \%$ nt identity with other reported P4 genomes, and PCVL258 and PCVL264 showed 100\% nt identity with previously reported genomes. To our knowledge, this is the first report on molecular characterization of porcine circoviruslike agents in dogs and cats. Further studies are needed to clarify the epidemiology, evolution, and pathogenesis of porcine circovirus-like agents in dogs and cats.
\end{abstract}

\section{Title: Porcine circovirus-like agents in sera of dogs and cats in China}

\section{Running title: porcine circovirus-like agents}

\author{
L. Wen*, A. Mao, J. Xie and K. He \\ Institute of Veterinary Medicine, Jiangsu Academy of Agricultural Sciences.Key Laboratory of Animal Di- \\ seases, Diagnostics, and Immunology, Ministry of Agriculture.Key Laboratory of Food Quality and Safety of \\ Jiangsu Province-State Key Laboratory Breeding Base, Nanjing, China
}

Jiangsu Co-innovation Center for Prevention and Control of Important Animal Infections Diseases and Zoonoses, Yangzhou, Jiangsu, China

* Corresponding author. Tel.: +86 2584390331.

E-mail address: wlbwh@hotmail.com

\section{Summary}

Porcine circovirus-like agents comprise two types of viruses: porcine circovirus-like viruses (P1, P2, P3, and P4) and porcine circovirus-like mini agents (PCVL258, PCVL264, PCVL201, and PCVL347). Of these, P1 has been identified in pigs, cattle, goats, and rabbits in China; P2, P3, P4, PCVL258, and PCVL264 have been identified in pigs; and PCVL201 and PCVL347 have been identified in cattle. The purpose of this study was to determine whether dogs and cats have been exposed to porcine circovirus-like agents. We screened 158 serum samples from diseased dogs and 41 from cats in China by PCR and nucleotide sequencing. In dogs, approximately $18 \%(n=28)$ were positive for P1, $17 \%(n=26)$ for PCVL258, and 9\% $(n=14)$ for PCVL264; in 
cats, $17.1 \%(n=7)$ were positive for $\mathrm{P} 1,9.8 \%(\mathrm{n}=4)$ for $\mathrm{P} 4$, and $14.6 \%(\mathrm{n}=6)$ for PCVL258. The P1 genomes in this study consisted of 648 nucleotides (nt), and shared 96.8 to $100 \%$ nt identity with other P1 genomes in GenBank. The P4 genome shared 98.3 to $100 \%$ nt identity with other reported P4 genomes, and PCVL258 and PCVL264 showed 100\% nt identity with previously reported genomes. To our knowledge, this is the first report on molecular characterization of porcine circovirus-like agents in dogs and cats. Further studies are needed to clarify the epidemiology, evolution, and pathogenesis of porcine circovirus-like agents in dogs and cats.

\section{KEYWORDS}

porcine circovirus-like agents, molecular identification, dog, cat

\section{INTRODUCTION}

Porcine circoviruses (PCVs) are small, non-enveloped DNA viruses belonging to the Circoviridae family. The PCVs genomes are single-stranded circular DNA, 1,700 to 2,000 nucleotides (nt) long. At present, PCV can be classified into four types: PCV1, PCV2, PCV3, and PCV4. PCV1 is considered to have no pathogenicity in pigs (Tischer et al., 1986), whereas PCV2 has been considered to be associated with PCV2-associated diseases (PCVAD), which cause huge economic losses to the swine industry worldwide (Harding \& Clark, 1998). Recently, PCV3 was identified in pigs with cardiac and multi-systemic inflammation, and in pigs with clinical signs of porcine dermatitis and nephropathy syndrome (PDNS) in the United States (Phan et al., 2016; Palinski et al., 2017). PCV4 was recently identified in pigs with severe clinical disease in China (Zhang et al., 2019).

Porcine circovirus-like agents are agents with truncated PCV2 genomes, 200 to 1,000 nt long, containing extraneous nucleotide sequences. At present, these agents can be divided into two types: porcine circovirus-like viruses and porcine circovirus-like mini agents. The former comprises four viruses, tentatively named P1, P2, ZJ-R (P3), and P4, whose genomes can encode virus proteins (Wen et al., 2008, 2012, 2015, 2018a). The latter also includes four agents, tentatively named PCVL258, PCVL264, PCVL201, and PCVL347, whose genomes have no ability to encode a protein (Wen et al., 2019). The detection rate of porcine circovirus-like virus P1 is significantly higher than that of other porcine circovirus-like agents, so more research has been conducted on $\mathrm{P} 1$. Genomic sequence analysis has revealed that the novel P1 virus possesses a single-stranded circular DNA genome of 648 nt containing eight open reading frames (ORFs) (Wen et al., 2014). It has higher homology with the partial Cap of a PCV2 strain except for 16 consecutive nucleotides (CGTTACTAGTGGATCC). The P1 capsid protein (Cap) encoded by ORF1 contains 114 amino acid (aa) residues, 120-121 aa fewer than PCV2. To date, two types of P1 virus have been identified, which are 648 and 647 nt long (based on the whole genome); 114 and 122 aa (based on the full-length capsid), respectively (Wen et al., 2017, 2018c). All porcine circovirus-like agents except for PCVL201 and PCVL347 were originally detected in diseased pigs in China. More recently, P1 DNA has also been detected in cattle, goats, and rabbits (Wen et al., 2018b). The goal of this study was to investigate the presence of porcine circovirus-like agents in dogs and cats in China.

\section{MATERIALS AND METHODS}

To detect porcine circovirus-like agents in dogs and cats, we collected 199 clinical serum samples from dogs $(n=158)$ and cats $(n=41)$ with clinical signs of respiratory tract or digestive tract disease from veterinary hospitals in the cities of Nanjing (Jiangsu province, China) and Shijiazhuang (Hebei province, China) between January 2018 and December 2019. Samples were stored at -80degC before analysis. DNA extraction and PCR amplification were performed as previously described (Wen et al., 2019). Briefly, total DNA from $200 \mu \mathrm{L}$ of serum was extracted using a DNA kit (Tiandz, Inc., China) according to the manufacturer's instructions. Two sets of back-to-back PCR primers, described previously, were designed to amplify the complete genome sequences. PCR was carried out using the Mix (green) (TsingKe Biotech Co., Ltd. Beijing, China) following manufacturer's instructions. After an initial denaturation at $98{ }^{\circ} \mathrm{C}$ for $2 \mathrm{~min}$, the PCR conditions were 40 cycles at $98{ }^{\circ} \mathrm{C}$ for $15 \mathrm{~s}, 62{ }^{\circ} \mathrm{C}$ for $15 \mathrm{~s}$, and $72{ }^{\circ} \mathrm{C}$ for $10 \mathrm{~s}$, followed by a final extension at $72{ }^{\circ} \mathrm{C}$ for 5 min. The PCR products were purified using a Gel Extraction Kit (AXYGEN, China) according to the manufacturer's instructions, then cloned into the pMD18T vector (TaKaRa, Dalian, China), and sequenced. In addition, all 
samples were screened for PCV2, PCV3, and PCV4 by conventional PCR using reference primers (Wen et al., 2012; Palinski et al., 2017; Zhang et al., 2019).

The resultant sequences were analyzed using DNAStar software version 7.10 (Lasergene). The complete genome sequences of porcine circovirus-like agents in this study and reference strains were aligned using the Clustal X program of DNAStar to determine nucleotide sequence similarities. Based on complete genome sequences, a phylogenetic tree was constructed using the neighbor-joining method in MEGA7.0 (http://www.megasoftware.net), using $p$-distances with 1,000 bootstrap replicates. We used 12 representative P1 strains for phylogenetic analysis, including 2 cattle-origin P1 strains (GenBank KY462783, KY462784), 2 rabbit-origin P1 strains (GenBank KY462785, KY462786), 2 goat-origin P1 strains (GenBank KY462787, KY462788), and 6 pig-origin P1 strains [GenBank EF514716 (the prototype sequence), JN040279, KU243695, KU323639 (the prototype elongated ORF1 sequence), KU324511, and KU356939]. In addition, we also used all other related porcine circovirus-like agents available on GenBank for analysis.

\section{RESULTS AND DISCUSSION}

Of the 158 tested serum samples from dogs, $17.7 \%(n=28)$ were positive for P1, 16.5\% $(n=26)$ for PCVL258, and $8.9 \%(\mathrm{n}=14)$ for PCVL264 based on PCR. In 41 samples from cats, $17.1 \%(\mathrm{n}=7)$ were positive for P1, 9.8\% $(\mathrm{n}=4)$ for $\mathrm{P} 4$, and $14.6 \%(\mathrm{n}=6)$ for PCVL258. No PCV2, PCV3, and PCV4 strains were identified in the PCR assays (data not shown). The results also revealed a co-infection of P4 and PCVL258 in a cat.

To further study the molecular epidemiology of these agents, we obtained the full-length genomic sequences from 18 strains, representing 4 P1, 3 PCVL258, and 2 PCVL264 strains in dogs, and 5 P1, 2 P4, and 2 PCVL258 strains in cats, all of which have been deposited in GenBank (accession nos. MH379143, MH379144, MT318811-MT318826). All 9 P1 viruses in this study had a circular genome length of 648 nt and shared 96.8 to $100 \%$ nucleotide identity with other available P1 genomes in GenBank. Compared with the nucleotide sequence of the prototype P1 strain (GenBank EF514716), the dog-origin P1 strain NJ01 (MH379143) and the cat-origin P1 strain NJ03 (MT318822) were similar to the prototype elongated ORF1 strain (KU323639) and had a nucleotide substitution at position 77 ( $\mathrm{T}$ to $\mathrm{C}$ ), which would result in a codon shift of the putative ORF1 starting at nt position 419 and a stop codon at aa position 122 . The ORF1 sequence of other strains in this study had a stop codon at aa position 114. Alignment of the whole genomic sequences of the $21 \mathrm{P} 1$ strains showed that there were multiple point or region mutations, for example, variations in 6 positions appeared specific for the cat-origin strain HeB02 (MT318824), including residues 331 (A to T), 337 ( $\mathrm{T}$ to $\mathrm{C}$ ), 344 ( $\mathrm{C}$ to $\mathrm{T}$ ), 371 ( $\mathrm{T}$ to $\mathrm{G}$ ), 376 ( $\mathrm{G}$ to $\mathrm{T}$ ), and 379-383 (CCCCC to TTGTT). The 2 P4 cat-origin strains NJ01 and NJ02 had a genome length of 710 nt. The nucleotide sequence of the cat-origin strain NJ01 was identical to that of NJ02. The overall nucleotide identity of strains NJ01 and NJ02 in the whole genome to other pig-origin strains ranged from 98.3\% (MF802838) to 100\% (MF802836). Whether of pig-origin or not, all PCVL258 and PCVL264 sequences were identical.

A corresponding phylogenetic tree (including 12 reference P1 sequences, 2 reference P4 sequences, 1 reference PCVL258 sequence, and 1 reference PCVL264 sequence) was constructed based on the full-length sequence. Phylogenetic analysis suggested that all P1 sequences from this study were scattered within the P1 viral lineage except one cat-origin strain (MT318824), the sequence of which showed more variation and was located in a separate branch. All P1 strains in this study were more closely related to other PCVL258 and PCVL264 strains than to P4 strains (Figure 1).

PCV2 is responsible for several multifactorial disease syndromes (especially for postweaning multisystem wasting syndrome (PMWS) in pigs. Furthermore, PCV2 can be transmitted across species barriers, and may cause severe fatal disease in other species (such as mink) (Wang et al., 2016). In addition, porcine circovirus-like agents, newly emerging causative agents in pigs, have a close evolutionary relationship with PCV2, based on phylogenetic analysis of genomic DNA sequences. The P1 viruses detected in dogs and cats in our study had high homology with P1 strains from pigs, goats, cattle, and rabbits.

\section{CONCLUSION}


In summary, we have confirmed, for the first time, the presence of porcine circovirus-like agents in dogs and cats with various clinical signs in China. Further studies will be required to ascertain whether these agents cause disease in dogs and cats and to determine the ecology and cross-species transmission mechanisms of these agents.

\section{ACKNOWLEDGEMENTS}

This study was supported by grants from National Key Research and Development Program (2018YFD0500101), and the National Natural Science Foundation of China (nos. 30972184, 31272574).

\section{CONFLICT OF INTEREST}

The authors declare that they have no conflict of interest.

\section{AVAILABILITY OF DATA AND MATERIALS}

The data set supporting the conclusions of this article is available in the GenBank.

\section{ETHICAL STATEMENTS}

This article does not contain any studies with human participants or animals performed by any of the authors.

\section{References}

Harding, J., \& Clark, E. (1998). Recognizing and diagnosing postweaning multisystemic wasting syndrome (PMWS). Swine Health and Production , 5, 201-203.

Palinski, R., Pineyro, P., Shang, P., Yuan, F., Guo, R., Fang, Y., .. Hause, B. M. (2017). Novel porcine circovirus distantly related to known circoviruses is associated with porcine dermatitis and nephropathy syndrome and reproductive failure. Journal of Virology, 91, e01879-16.

Phan, T. G., Giannitti, F., Rossow, S., Marthaler, D., Knutson, T., Li, L., ... Delwart, E. (2016). Detection of a novel circovirus PCV3 in pigs with cardiac and multi-systemic inflammation. Virology Journal,13, 184.

Tischer, I., Mields, W., Wolff, D., Vagt, M., \& Griem, W. (1986). Studies on epidemiology and pathogenicity of porcine circovirus. Archives of Virology, 91, 271-276.

Wang, G. S., Sun, N., Tian, F. L., Wen, Y. J., Xu, C., Li, J., Chen, Q. \& Wang, J. B. (2016). Genetic analysis of porcine circovirus type 2 from dead minks. The Journal of General Virology , 97, 2316-2322.

Wen, L., He, K., Yu, Z., Mao, A., Ni, Y., Zhang, X., .. Lv, L. (2012). Complete genome sequence of a novel porcine circovirus-like agent. Journal of Virology, 86, 639.

Wen, L., He, K., Yang, H., Ni, Y., Zhang, X., Guo, R., \& Pan, Q. (2008). Complete nucleotide sequence of a novel porcine circovirus-like agent and its infectivity in vitro.Science in China. Series C, Life Sciences, 51(5), 453-458.

Wen, L., Wang, F., He, K., Li, B., Wang, X., Guo, R., \& Xie, J. (2015). Recombination in truncated genome sequences of porcine circovirus type 2.Archives of Virology, 160(1), 371-374.

Wen, L., Mao, A., Zhu, X., Xie, J., \& He, K. (2018a). Detection of a novel porcine circoviruslike agent in aborted pig foetuses. Transboundary Emerging Diseases , 65(5), 1364-1366.

Wen, L., Mao, A., Xie, J., \& He, K. (2019). Complete genome sequences of porcine-circoviruslike mini agents in pigs and cattle.Archives of Virology, 164(2), 633-635.

Wen, L., Jiao, F., Zhang, D., Mao, A., Liu, C., Xie, J. \& He, K. (2017). Genome sequence of a porcine circovirus-like virus P1 mutant in China. Archives of Virology, 162, 585-586.

Wen, L., Mao, A., Fan, Z., Li, W., Xiao, Q., Liu, Q., .. He, K. (2018b). Porcine circovirus-like virus P1 in cattle, goats and rabbits in China. Transboundary Emerging Diseases, 65, e217-e218. 
Wen, L., Mao, A., Jiao, F., Zhan, D., Xie, J. \& He, K. (2018c). Detection of porcine circovirus-like virus P1 in Hebei, China.Transboundary Emerging Diseases, 65, 1133-1136.

Wen, L., Wang, F., He, K., Li B, Wang, X., Guo, R. \& Xie, J. (2014). Transcriptional analysis of porcine circovirus-like virus P1. BMC Veterinary Research, 10, 287.

Zhang, H. H., Hu, W. Q., Li, J. Y., Liu, T. N., Zhou, J. Y., Opriessnig, T., \& Xiao, C. T. (2019). Novel circovirus species identified in farmed pigs designated as Porcine circovirus 4, Hunan province, China.Transboundary Emerging Diseases, doi: 10.1111/tbed.13446

\section{FIGURE LEGEND}

Figure 1. Phylogenetic analysis of the complete nucleotide sequences of porcine circovirus-like agents. The phylogenetic tree was constructed using the neighbor-joining method and the $p$-distance model with statistical analysis supported by bootstrapping with 1,000 replicates. Bootstrap values $<50 \%$ are not shown. The scale bar represents number of nucleotide substitutions per site. The porcine circovirus-like agents of dog and cat origin detected in this study are marked with black triangles. 


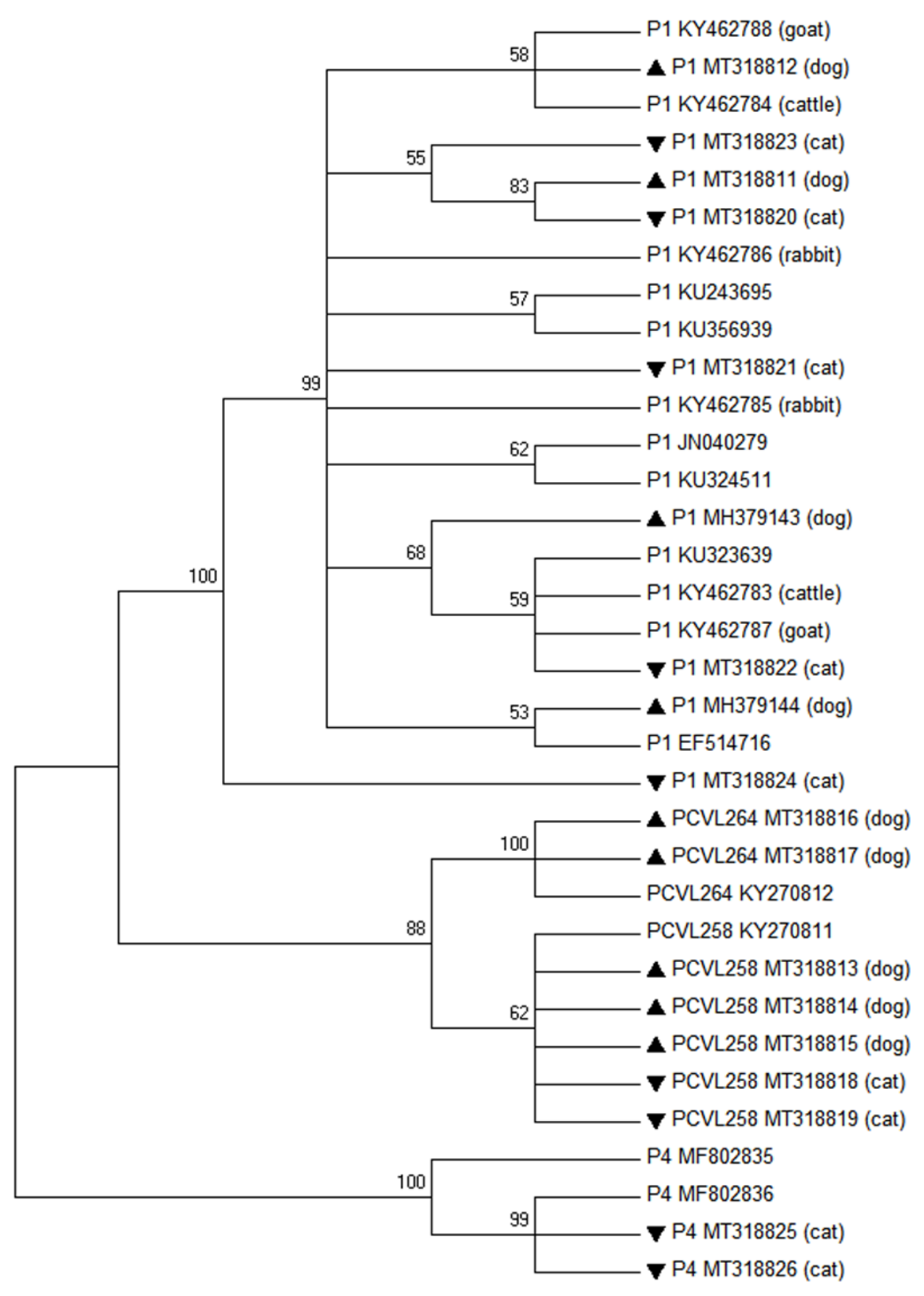

